\title{
Safe Doses and Cancer Treatment Evaluation
}

\author{
Emad Y. Moawad \\ Faculty of Engineering, Ain shams university, Cairo, Egypt \\ *Corresponding Author: emadmoawad@hotmail.com
}

Copyright $(2013$ Horizon Research Publishing All rights reserved.

\begin{abstract}
The aim of this research is to check the efficacy of radiotherapy after execution that helps in preserving patients' rights against the randomized dose that settled statistically and assessed in standard models ignoring patient-specific factors. Based on studying a dose-response relationship, a mathematical model is presented describes the initial tumor energy (E0Tumor) prior therapy after treatment execution -even if it was not predetermined- by monitoring the tumor response along the treatment phases and compared to the applied dose energy (E0Dose). Our model allows mechanic risk predictions to be made at high radiotherapeutic doses as well as at low doses, besides to the second cancer risk prevention. Thus, the administered dose errors could be determined and consequently preserving patients' rights to evaluate the cancer treatment through the provided mathematical model. Reasons of tumor regrowth are either underestimation or overestimation of the administered dose; the safe dose of the successful treatment occurs only in the case of: E0Dose = E0Tumor, where tumor regrowth energy in such a case would be vanished. Dose assessment by ignoring patient-specific factors and using standard models is responsible for wide range of doses that lead to tumor regrowth and second cancer risks. Current approach suggests settling down a new protocol for the proper ranges of radionuclide doses based on a personalized staging system.
\end{abstract}

Keywords Safe Doses; Emad Formula; Tumor Growth Energy

\section{Introduction}

Estimates of radiation carcinogenesis after radiotherapy based on epidemiological studies of patients treated with old techniques [1] have concluded such risks but haven't provided a correlation of cancer induction with dose yet. Thereby, radiotherapy is considered a potential cause of cancer, since secondary malignancies are seen in a very small minority of patients during phase III afterwards clinical trials generally many years after they have received a course of radiation treatment [2-4]. Accordingly, survivors who are treated with radiation have an increased risk of certain second cancers, so they should get careful follow-up. The ultimate goal of new cancer therapies is cure; this goal although sometimes achieved in hematological malignancies, has rarely been achieved in disseminated solid cancers. A good cancer treatment should ideally prolong survival while preserving a high quality of life cost-effectively, which suggests the necessity for long-term follow up. Nevertheless prolonged survival in a clinical trial in some more slowly progressing cancers can take 5-10 years or longer [5]. Such trials are expensive, not only in cost but in time. As a consequence of the better current available radiation treatment modalities, cancer cure rates have been increased [6]. As a result, there are now many long term survivors of cancer who are at risk of late effects of therapy, including secondary cancers. In the vast majority of cases, this risk is greatly outweighed by the reduction in risk conferred by treating the primary cancer [7]. The risk of second cancers must always be weighed against the benefits gained with treatment. The risks of treatments should always be compared carefully against the cost of not using such treatments. It is therefore important to note that in breast cancer the benefits of radiotherapy still very much outweigh the risks of developing subsequent second cancers. For many new cancer treatments, the long-term effects that cause second cancers are not yet known. The need for ongoing follow-up of cancer survivors is important so that we can better understand the long-term effects of cancer treatments. Kendal et al. [8] go on to argue that this is associated with uncertainties, as there is no good evidence that prostate radiotherapy produces any detectable increase in the risk of rectal cancer, despite the wide range of doses, from low to very high, to which the rectum is typically exposed during external-beam prostate radiotherapy $[9,10]$, and concluded that the differences in rectal cancer risks between the different treatment groups, including the increase for radiotherapy relative to surgery, must be due to some unknown cause and not to radiation!. Tubiana $M$ suggested that some radiotherapy-induced second cancers can be the consequence of late normal tissue damage leading to a precancerous lesion [11]. Such arguments negate the observational evidence for radiation-induced cancer in humans; some patients are not successfully treated initially, resulting in relapse and less frequently death, of equal importance, many patients may receive more therapy than needed [12]. This is particularly true for patients with stage III disease [13, 14]. In fact there is good evidence that 
radiotherapy produces a detectable increase in the risk of a second cancer, for the wide range of doses, from low applied in Low Irradiated Dose Treatment (LIDT) to very high applied in Over Irradiated Dose Treatment (OIDT), estimated statistically to which inducing the second cancer is typically during the radiotherapy $[15,16]$. But, is it true that radiotherapy does not measurably increase the risk of secondary cancer? Unfortunately, if a dose correlation is deduced, cancer induction is usually related to integral dose or average organ dose and thus implies a linear dose-response relationship. Thus, such data cannot be used directly to obtain non-linear dose-response relationships for radiotherapy. Therefore, as an alternative, cancer risk models can be used to estimate second malignancies after radiotherapy. Those models can be validated with physical studies. Moawad showed through his physical approach that the accuracy of estimating the initial effective radioactive dose depends on the equivalence of the initial growth energy of the tumor (E0.T) and the decay energy of the effective radioactive dose (E0.D), which is known by strategy of administering the required dose according to Work-Energy Principle Treatment (WEPT) [17]. Current thesis aims to evaluate the administered doses of some already executed cancer treatments to clarify the methodology of preserving patients' rights against the randomized doses that were settled statistically and assessed in standard models which ignore subject specific factors based on studying a dose-response relationship along the treatment phases.

\section{Method and Materials}

Evaluating the administered doses of the already executed cancer treatments can be done by determining the accumulated difference of the tumor and the radionuclide energies along the treatment based on studying a dose-response relationship by monitoring the mechanism of the tumor response along the treatment phases. Then the initial tumor energy (E0Tumor) prior therapy can be derived after treatment execution provided that it wasn't predetermined to be comparable to the administered dose energy (E0Dose), according to the following mathematical model:

$$
E_{0 . \text { Tumor }}=E_{0 . \text { Dose }} \div\left(\int_{0}^{T} e^{\frac{-\ln 2 \times t}{t_{1 / 2 \text { Radionuclide }}}} d t-\int_{0}^{T} e^{\frac{-\ln 2 \times t}{t_{1 / 2 \text { Tumor Shrinkage }}}} d t+1\right)
$$

Eqt 1.

Provided that the equivalence of dose and tumor initial energies i.e. E0Dose $=$ E0Tumor enables tumor regrowth energy in such case to be vanished, where the accumulated energies of each of the tumor shrinkage and the decayed radionuclide along the treatment would be equal as

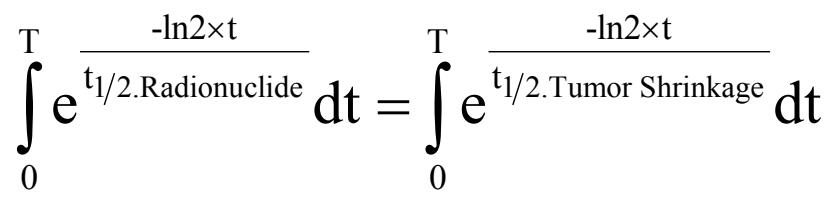

Evaluating E0Dose from the tumor response by determining E0Tumor after treatment execution:

This application for checking the efficacy of radiotherapies after their execution, which helps in preserving patients rights against the randomized statistical dose assessment which ignore patient specific factors. It shows that as the tumor sizes and their doubling time in patients varied widely, as these differences produce significant differences between doses assessed physically even for the same sizes [14]. Barendswaard et al. [15] showed that four- to 6-wk-old athymic female Swiss (nu/nu) mice (body weight, 20-25 g) from their in-house nude mouse facility were injected with $10 \times 10^{6} \mathrm{SW} 1222$ cells in the left thigh muscle. After 5-7 d, mice bearing tumors of 140-900 $\mathrm{mg}$ were selected. A total of 169 mice were divided into groups of 4-9 mice. Fourteen groups were administered varying amounts of mAb A33 labeled with 131I. The activities of 131I-A33, activities ranged from 0.925 to 18.5 $\mathrm{MBq}(0.025-0.5 \mathrm{mCi})$. Tumor size was measured bidimensionally with calipers, and the volume was calculated assuming elliptic geometry. Initial tumor sizes were between 0.14 and $0.90 \mathrm{~cm}^{3}$ (mean, $0.44 \mathrm{~cm}^{3}$ ). Mice with tumors of differing sizes were divided into each group such that the tumor size spectrum for each group was similar. The tumors were measured every 3 or $4 \mathrm{~d}$ for $100 \mathrm{~d}$ or until the death of the animal. Mice were killed when the tumor caused apparent discomfort in walking or when its volume exceeded $2 \mathrm{~cm}^{3}$. Observations showed that tumor growth was retarded after treatment to an extent that was dependent on the amount of activity administered. Barendswaard et al. showed that Tumors were considered cured if they failed to regrow over the period of observation ( $100 \mathrm{~d}$ after treatment) [15]. Occasional tumor cures were seen at intermediate administered activities of 131I (3.7-11.1 MBq) that represent WEPT, but a higher value $(14.8 \mathrm{MBq})$ that represents OIDT did not produce any cures. Four of 5 tumors in this group became temporarily undetectable but subsequently recurred between day 40 and day 80 . While the highest activity of $131 \mathrm{I}$ administered $(18.5 \mathrm{MBq}=0.5 \mathrm{mCi})$ that represents not only OIDT but also the maximum tolerated activities of 131I in this model system resulted in tumor cures in all 4 animals in that group. Activities of 18.5 $\mathrm{MBq} 131 \mathrm{I}$ caused petechiae, which became apparent after 2 $\mathrm{d}$ and confluent after $4 \mathrm{~d}$, as well as progressive weight loss. Median tumor energy, normalized to initial energy, as function of time in nude mice bearing SW1222 xenografts during therapy shown in figure 1. 


\section{Dose-Tumor Response Relationship}

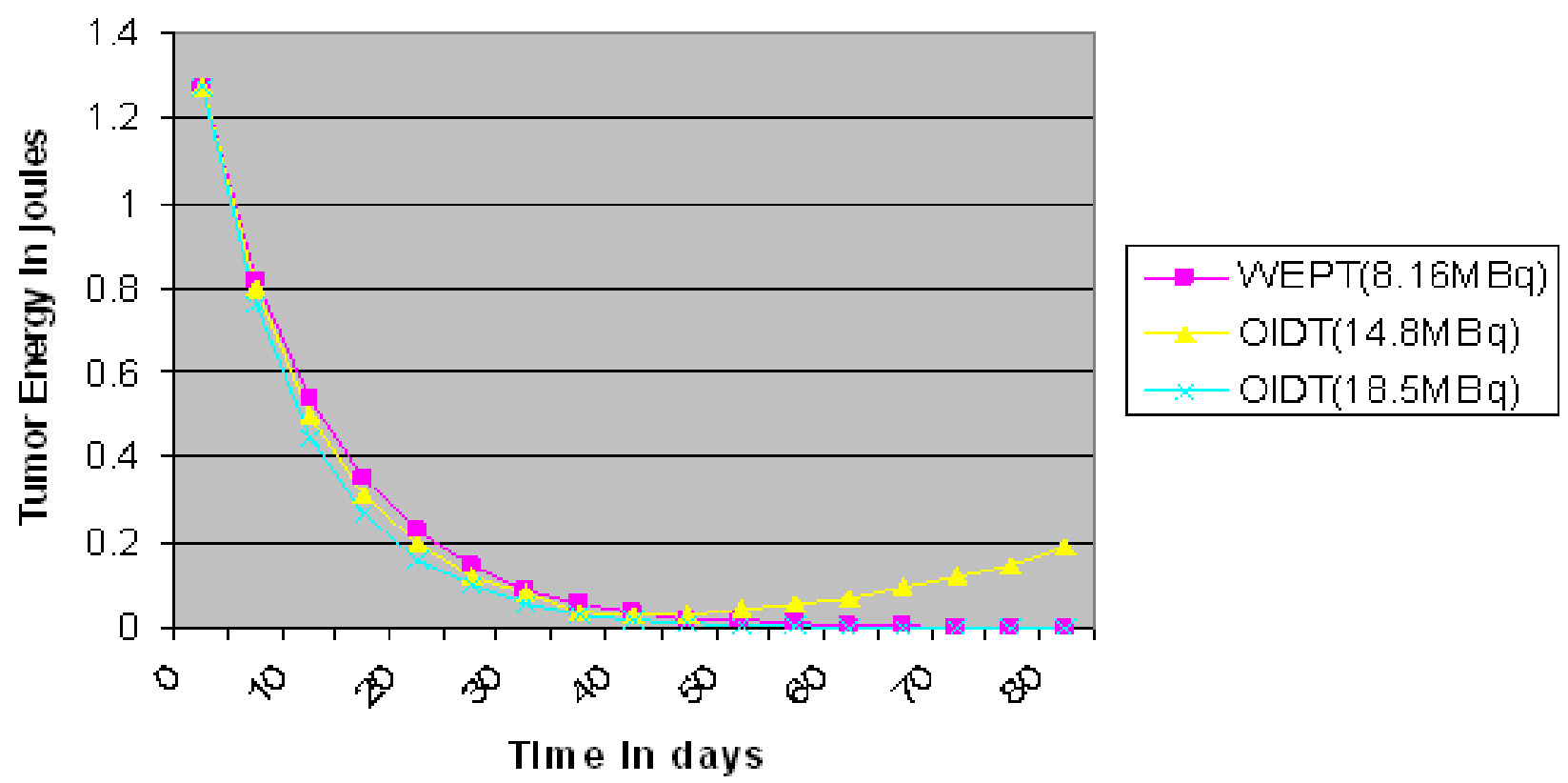

Figure 1. shows a dose-response relationship for the different administered doses. The highest activity of $131 \mathrm{I}$ administered $(18.5 \mathrm{MBq}=2.88 \mathrm{~J})$ resulted in tumor cures in all animals, but the value of $(14.8 \mathrm{MBq}=2.305 \mathrm{~J})$ did not produce any cures and tumor regrow was between day 40 and day 80 . The tumor response after dose delivery followed an exponential shrinkage of half life time of $6.5 \mathrm{~d}$, continued only for the tumor that assigned to the $8.16 \mathrm{MBq}(1.2721$ J) or $18.5 \mathrm{MBq}$ only of $131 \mathrm{I}$ dose, and cured.

\section{Results}

A dose-response relationship has been studied for the different administered doses. The highest activity of 131I administered $(18.5 \mathrm{MBq})$ resulted in tumor cures in all animals, but the value of (14.8 MBq) did not produce any cures and tumor regrow was between day 40 and day 80 . The tumor response after dose delivery followed an exponential shrinkage of half life time of $6.5 \mathrm{~d}$, continued only for the tumor that assigned to the $18.5 \mathrm{MBq}$ only of 131I dose, and cured. On the contrary, the tumor which has been assigned to the dose of $14.8 \mathrm{MBq}$ reached its minimum size after $40 \mathrm{~d}$; afterwards it relapsed, following an exponential growth of $14.46 \mathrm{~d}$ doubling time, and reached a relative tumor size of 6.8 with respect to the minimum size after $80 \mathrm{~d}$ from the dose delivery. As the initial tumor size $\left(m_{0}\right)$ was not provided by Barendswaard et al, the initial tumor energy (E0Tumor) prior the treatment can be derived after treatment execution from the thesis of the equivalence of the difference between areas under the curves of tumor response during each of WEPT\& OIDT, and the difference between drugs released energy and that of the tumor according to Eqt 1, i.e.

$$
E_{0 . T} \times\left(\int_{0}^{T} e^{\frac{-\ln 2 \times t}{t_{1} / 2 . \text { Isotope }}} d t-\int_{0}^{T} e^{\frac{-\ln 2 \times t}{t_{1} / 2 . \text { Shrinkage }}} d t\right)=E_{0 . D}-E_{0 . T}
$$

Then by substituting data of this experiment shown by Barendswaard et al [15](shrinkage half life time was $6.5 \mathrm{~d}$ along 20 days, for $18.5 \mathrm{MBq}$ initial dose activity of 131I which corresponds to $2.881 \mathrm{~J}$ ), calculation of the initial tumor energy shows that $\mathrm{E}_{0 . \mathrm{T}}=1.2721 \mathrm{~J}$, which corresponds to energy of a dose of $8.168 \mathrm{MBq}$ only. This means that the administered dose of Barendswaard et al treatment was supposed to be $8.168 \mathrm{MBq}$ in a WEPT. Consequently applying the higher doses of $14.8 \mathrm{MBq}$ and $18.5 \mathrm{MBq}$ are considered OIDTs. All treated tumors shrunk by a rate of $7.4 \mathrm{~d}$ caused the tumor energy to decrease from $1.2721 \mathrm{~J}$ to $0.028 \mathrm{~J}$ after 40 days as shown in figure 1. The initial tumor size $(\mathrm{m} 0)$ can be calculated to check the hypothesis of the current thesis by determining the energy of the tumor hypoxic cell (EHypoxic cell) using Emad formula as shown by Moawad as follows:

EHypoxic cell $=\ln \left(\ln \frac{\ln 2}{t_{D}}\right)^{2}$ Emad Eqt 2, where $t_{D}$ is the tumor doubling time, Emad $=23234.59 \mathrm{MeV}$ [17-19].

Accordingly, the malignant tumour fraction $(\% \mathrm{M})$ can be determined from the following equation model:

$$
\mathrm{M} \%=100 \% \text { for EHypoxic cell } \geq 5: 2 \text { Emad }
$$

$\mathrm{M} \%=79.53$ EHypoxic cell -313.94, EHypoxic cell $<5: 2 \mathrm{Emad}$ Eqt $3[18,19]$.

Thus, from the tumor response of the control group, the tumor doubling time was $4 \mathrm{~d}$, consequently from Eqt 2 the hypoxic cell growth energy was $E_{\text {Cell }}=\ln \left(\ln \frac{\ln 2}{4 \times 24 \times 60 \times 60}\right)^{2}$ $=5.14$ Emad, from Eqt 3, M\% is $94.84 \%$. Then, $m_{0}$ can be calculated as follows: The initial number of hypoxic cells 
$\left(\mathrm{C}_{\text {Hypoxic cell }}\right)$ which approximately represents $10 \%$ of the total number of tumor cells $[17,18]$ is

$$
\mathrm{C}_{\text {Hypoxic cell }}=\frac{\mathrm{E}_{0 \mathrm{~T}} ? .242 ? 0^{12} \mathrm{MeV}}{\mathrm{M} \% \times \mathrm{E}_{\text {Hypoxic cell }} ? 3234.59 \mathrm{MeV}}[18,19]
$$

$$
=\frac{1.2721 \times 6.242 \times 10^{12}}{0.9484 \times 5.14 \times 23234.59}=7.0 \times 10^{7} \text { hypoxic cells. }
$$

Thus the total number of tumor cells was $7 \times 10^{8}$ cells. By knowing that number of tumor cells/gm is about $10^{9}$ cells/gm [17], then $m_{0}$ was $0.7 \mathrm{gm}$ which is accepted as a median value for the set of treated tumors ranged from $0.14 \mathrm{~g}$ to $0.90 \mathrm{~g}$, as given by Barendswaard et al [15].

\section{Discussion}

The issue of radiotherapy related second cancers will become increasingly pertinent, where it is unquestionably the responsibility of the radiation oncology profession as a whole to induce second cancer [20]. With respect to ionizing radiation, the meaning of a safe dose or dose-rate is a dose or dose-rate at which all exposed persons are safe as the exposure occurs, and all are safe afterwards, no fraction will be killed later by radiation-induced cancer. Probably the most important issue in this field is whether or not there exists some low dose, or dose-rate, of low-LET ionizing radiation which produces no cancer at all in exposed populations. In short, is there a safe region below some threshold of danger? Paracelsus, the 16th century Swiss physician, once said, "All substances are poisons: there is none which is not a poison [21]. Consequently, it can be said that "no safe dose or dose-rate" means that no one is safe as the exposure occurs, and afterwards, some fraction of the exposed persons will die from radiation-induced cancer, and the rest will be safe from it. The right dose differentiates a poison and a remedy, where it can be evaluated as shown in Method and Materials section. The best fit of the presented model to the provided experimental data allows for optimization of radiotherapy protocols and preserving Patients' rights against administered dose errors, where reasons for tumors regrowth are either underestimation or overestimation the administered dose, safe doses of successful treatments occurs only in the case of: E0Dose $=$ E0Tumor, where tumor regrowth energy in such case would be vanished [22]. These results can be a reply for the preceded question "Is it true that radiotherapy does not measurably increase the risk of secondary cancer?" Moreover, this relation enables us to test all the background of medical dosimetry experiments that based on the statistical analysis, as well as prior successful treatments had been conducted in different schools of medicine. This can be an answer for puzzling observations on comparing radiotherapy treatments in different schools of medicine all over the world. Although one can find the same disease in nearly the same circumstances, but with completely different recurring doses, hitherto no-one has had a conceptual reasoning for these invariant doses. Although statisticians and physicians classify characteristics of patients more specifically, even whether white or black, they finally concluded that "dosimetry never inherits identical results! [23]" Several assumptions had to be made to simplify the biological processes leading to cancer induction; in general, all factors or agents - and radiation is one of them- that contribute in increasing the growth energy of the biological cell, are considered as cancer causes, this is first shown by Emad Moawad[ 24 ]. More specifically, agents that lead to the increase of the cell doubling time or in other words cause the cell to divide more slowly, if severe or prolonged it could lead to induce cancer $[25,26]$. Such effect occurs generally due to radiation exposure and hence radiotherapy, as this phenomenon was evident by several experimental observations. Such explanation reveals why the breast cancer radiotherapy can cause lung cancer [27, 28], and secondary rectal cancer rates are higher in prostate cancer patients [29, 30]. Since administered dose estimation techniques for internal radiation emitters continue to evolve as described for applying strategy of WEP in treatments, preventing serious toxicities to normal vital organs is not only essential but also, perhaps, the ethical responsibility of all involved in treating cancer patients to preserve patients rights against the randomized statistical dose assessment which ignore subject specific factors [31]. Such strategy can be achieved through accurate measurement of the uptake and retention of the radiopharmaceuticals in organs and tissues which becomes a challenging, where a reasonably accurate assessment of radionuclide pharmacokinetics is feasible with current imaging techniques $[17,32]$. An important goal of current model development is the second cancer risk prevention. Hereby this approach suggests that the equivalence of dose and tumor initial energies i.e. E0Dose $=$ E0Tumor enables tumor regrowth energy in such case to be vanished, where the accumulated energies of each of the tumor shrinkage and the decayed radionuclide along the treatment would be equal

$$
\text { i.e. } \int_{0}^{\mathrm{T}} \mathrm{e}^{\frac{-\ln 2 \times \mathrm{t}}{\mathrm{t}_{1 / 2 \text {.Isotope }}}} d \mathbf{t}=\int_{0}^{\mathrm{T}} \mathrm{e}^{\frac{-\ln 2 \times \mathrm{t}}{\mathrm{t}_{1 / 2 . \text { Shrinkage }}}} d \mathbf{t}
$$

Briefly, our model allows mechanic risk predictions to be made at high radiotherapeutic doses as well as at low doses, can track the time dependency of risk mechanically, and is qualitatively, quantitatively better at describing background tumor response and second cancer incidence for all radiotherapy stages, with the same number of adjustable parameters; if the accumulated energy of the decayed radionuclide was less than that of tumor shrinkage i.e.

$$
\left(\int_{0}^{\mathrm{T}} \mathrm{e}^{\frac{-\ln 2 \times \mathrm{t}}{\mathrm{t}_{1 / 2 . \text { Isotope }}}} \mathrm{dt}-\int_{0}^{\mathrm{T}} \mathrm{e}^{\frac{-\ln 2 \times \mathrm{t}}{\mathrm{t}_{1 / 2 . \text { Shrinkage }}}} \mathrm{dt}\right)<0
$$

the tumor growth energy wont be vanished as a result of underestimation of the administered dose, which means that dose energy is insufficient and less than the initial treated 
tumor energy i.e. E.0D $<$ E.0T[22]. Vice versa if the accumulated energy of the decayed radionuclide was greater than that of tumor shrinkage i.e.

$$
\left(\int_{0}^{\mathrm{T}} \mathrm{e}^{\frac{-\ln 2 \times \mathrm{t}}{\mathrm{t}_{1 / 2 \text {.Isotope }}}} \mathrm{dt}-\int_{0}^{\mathrm{T}} \mathrm{e}^{\frac{-\ln 2 \times \mathrm{t}}{\mathrm{t}_{1 / 2 . \text { Shrinkage }}}} \mathrm{dt}\right)>0
$$

the tumor growth energy would be decreased rapidly as a result of overestimation of the administered dose, which means that dose energy is greater than the initial treated tumor energy i.e. E.0D > E.0T[22]. This rapid decrease in volume and subsequent mass reduction result in an increase of mean absorbed dose to the tumor of as much as a factor of 1.75[33] which contributes in case of the higher administered dose than the safe one to increase the risks of tumor regrowth or to develop a second cancer[22]. The ability to reasonably predict cancer at both low and high radiation doses using a physically based mathematical model, incorporating formalisms for both short-term and long-term processes, should enable this model to be used for optimization of radiotherapy protocols, by introducing second cancer risk as an additional criterion. This underscores the importance of individual patient treatment planning where patients should be protected against both cases by settling down a new protocol to administer the right dose that is suitable to Patient-specific factors. In addition, such strategy enables more accurate safe dose calculations as differences between use of standard models and patient-specific factors are considerable of very wide ranges.

\section{Conclusions}

Despite it is interesting to make dosimetry direct and easy, dosimetry should be patient specific for more accurate safe dose calculations. Statistically- modulated radiotherapy may result in a higher incidence of tumor regrowth or second primary cancers than that caused by the physically-conventional radiotherapy. As is usually the case, current thesis suggests approving new histogram for Dose-Tumor Energy to settle down a new protocol for cancer treatments.

\section{Conflict of Interest}

The author declares that there is no conflict of interest concerning this paper.

\section{REFERENCES}

[1] Boice JD, Land CE, Preston DL (1996) Ionizing radiation. In Cancer Epidemiology and Prevention, Schottenfeld D and
Fraumeni JF (eds) pp 319-341, Oxford University Press: New York

[2] Brenner DJ, Curtis RE, Hall EJ, Ron E (2000) Second malignancies in prostate carcinoma patients after radiotherapy compared with surgery. Cancer 88:398-406

[3] Ron E (2006) Childhood cancer-treatment at a cost. J Natl Cancer Inst 98:1510-1511

[4] Harlan LC, Potosky A, Gilliland FD, et al. Factors associated with initial therapy for clinically localized prostate cancer: Prostate cancer outcomes study. J Natl Cancer Inst 2001;93:1864-1871.

[5] Curtis RE, Boice Jr JD, Stovall M, Bernstein L, Greenberg RS, Flannery JT, Schwartz AG, Weyer P, Moloney WC, Hoover RN (1992) Risk of leukemia after chemotherapy and radiation treatment for breast cancer. N Engl J Med 326: $1745-1751$

[6] Brenner DJ, Shuryak I, Russo S, Sachs RK (2007) Reducing second breast cancers: a potential role for prophylactic mammary irradiation. J Clin Oncol 25:4868-4872

[7] Uwe Schneider, Jürgen Besserer, Hypofractionated radiotherapy has the potential for second cancer reduction, Theoretical Biology and Medical Modelling 2010, 7:4

[8] Kendal WS, Eapen L, MacRae R, et al. Prostatic irradiation is not associated with any measurable increase in the risk of subsequent rectal cancer. Int $\mathrm{J}$ Radiat Biol Oncol Phys 2006; 65:661- 668.

[9] Pollack A, Zagars GK, Starkschall G, et al. Conventional vs. conformal radiotherapy for prostate cancer: Preliminary results of dosimetry and acute toxicity. Int $\mathrm{J}$ Radiat Oncol Biol Phys 1996;34:555-564.

[10] Lee CT, Dong L, Ahamad AW, et al. Comparison of treatment volumes and techniques in prostate cancer radiation therapy. Am J Clin Oncol 2005; 28:618-625.

[11] Tubiana M: Can we reduce the incidence of second primary malignancies occurring after radiotherapy?A critical review.Radiother Oncol 2009,91(1):4-15.

[12] Breslow NE, Ou SS, Beckwith JB, et al. Doxorubicin for favorable histology, stage II-IIIWilms tumor: results from the NationalWilmsTumor Studies. Cancer 2004; 101:1072^ 80 .

[13] Grundy RG, Hutton C, Middleton H, et al. Outcome of patients with stage III or inoperableWT treated on the second United KingdomWT protocol (UKWT2); a United Kingdom Children's Cancer Study Group (UKCCSG) study. Pediatr Blood Cancer 2004;42: 311^9

[14] Pollack A, Zagars GK, Starkschall G, et al. Conventional vs. conformal radiotherapy for prostate cancer: Preliminary results of dosimetry and acute toxicity. Int $\mathrm{J}$ Radiat Oncol Biol Phys 1996; 34:555-564.

[15] Barendswaard EC, Humm JL, O’Donoghue JA et al (2001) Relative therapeutic efficacy of 125I- and 131I-labeled monoclonal antibody A33 in a human colon cancer xenograft. J Nucl Med 42:1251-1256, Abstract/Free Full Text

[16] Lee CT, Dong L, Ahamad AW, et al. Comparison of treatment volumes and techniques in prostate cancer 
radiation therapy. Am J Clin Oncol 2005; 28:618-625.

[17] Emad Moawad. Isolated System Towards A Successful Radiotherapy Treatment, Nuclear Medicine and Molecular Imaging (2010) 44:123-136

[18] Emad Y. Moawad, Clinical and pathological staging of the cancer at the nanoscale, Cancer Nano (2012) 3:37-46

[19] Emad Y. Moawad, Reconciliation between the clinical and pathological staging of cancer, Comparative Clinical Pathology (2012) (DOI: 10.1007/s00580-012-1603-6)

[20] Gold DG, Neglia JP, Dusenbery KE. Second neoplasms after megavoltage radiation for pediatric tumors. Cancer 2003;97:2588-2596.

[21] Paracelsus. Four Treatises of Theophrastus von Hohenheim Called Paracelsus. Sigerist $\mathrm{H}$ et al., trans. Baltimore, MD: Johns Hopkins University Press; 1996. [Paracelsus is the author; Sigerist is the translater.]

[22] Emad Y. Moawad Radiotherapy and risks of tumor regrowth or inducing second cancer. Cancer Nanotechnology (2011) 2:81-93

[23] Katherine K. Matthay, Colleen Panina, Correlation of Tumor and Whole-Body Dosimetry with Tumor Response and Toxicity in Refractory Neuroblastoma Treated with 131I-MIBG, Journal of Nuclear Medicine Vol. 42 No. 11 $1713-1721$

[24] Emad Y. Moawad, Mechanism of Nuclear transmutations in the Biological Culture .MPM-D.3 15:30 HPS 2011 Annual Meeting hpschapters.org/2011AM/program/singlesession.php3?sessid $=$ MPM-D

[25] Hypoxia and adaptive landscapes in the evolution of carcinogenesis; R.J. Gillies and R.A. Gatenby; Cancer Metastiasis Rev. 26: 1573, 2007. \&-Hypoxia-driven selection of the metastatic phenotype; R. Sullivan and C.H. Graham; Cancer Metastasis Rev. 26: 319, 2007.

[26] Brown JM. The hypoxic cell: a target for selective cancer therapy-Eighteenth Bruce F. Cain Memorial Award lecture. Cancer Res. 1999;59:5863-5870.

[27] R Roychoudhuri, H Evans, Radiation-induced malignancies following radiotherapy for breast cancer, British Journal of Cancer (2004) 91, $868-872$

[28] Smith RE, Bryant J, DeCillis A, Anderson S (2003) Acute myeloid leukemia and myelodysplastic syndrome after doxorubicin-cyclophosphamide adjuvant therapy for operable breast cancer: The National Surgical Adjuvant Breast and Bowel Project experience. J Clin Oncol 21: 1195- 1204

[29] Trachette L. Jackson A Mathematical Investigation of the Multiple Pathways to Recurrent Prostate Cancer: Comparison with Experimental Data Neoplasia . Vol. X, No. Y, 2004 DOI 10.1593/neo.04259

[30] David J. Brenner, D.SC. , INDUCED SECOND CANCERS AFTER PROSTATE-CANCER RADIOTHERAPY: NO CAUSE FOR CONCERN? Int. J. Radiation Oncology Biol. Phys., Vol. 65, No. 3, pp. 637-639, 2006

[31] Stabin MG. Internal dosimetry in the use of radiopharmaceuticals in therapy: science at a crossroads?
Cancer Biother Radiopharm. 1999; 14:81 89.[Medline]

[32] Rajendran JG, Fisher DR, Gopal AK, Durack LD, Press OW, Eary JF. High-dose 131I-tositumomab (anti-CD20) radioimmunotherapy for non-Hodgkin's lymphoma: adjusting radiation absorbed dose to actual organ volumes. $\mathrm{J}$ Nucl Med. 2004;45:1059-1064.[Abstract/Free Full Text]

[33] Hindorf C, Linden O, Stenberg L, Tennvall J, Strand SE (2003) Change in tumor-absorbed dose due to decrease in mass during fractionated radioimmunotherapy in lymphoma patients. Clin Cancer Res 9:4003S-4006S 